\title{
Beitrag zur vergleichenden Anatomie und Physiologie des Herzens.
}

\author{
Von
}

Joh. Dogiel.

Hierzu Tafel XII und XIII und 3 Textfiguren.

Trotz aller Schwierigkeiten, welche bei der Erforschung des Baues und der Function des Herzens zu überwinden sind, beschäftigen sich doch hin und wieder recht Viele mit der Herbeischaffung des nöthigen Materials zu einem vollendeten und soliden, wenn auch in weiter Zukunft zu Stande kommenden Bau, der uns über das Wesen der Lebensäusserungen des menschlichen Herzens Aufschluss zu ertheilen vermag. Ueberzengt, dass hierbei jeder neue, zuverlässige, wenn auch noch so winziger Baustein nicht unbeachtet bleiben kann, liefere ich den sich für diese Frage Interessirenden die vorliegende kurze Notiz als Material zu dem oben erwähnten Bau, wenn auch dieses Material noch so gering sein möge.

Noch gegenwärtig wünschen einige Physiologen sich von der Richtigkeit der von A. Haller ${ }^{1}$ ) stammenden Behauptung, dass die durch das Blut stimmulirte Herzmuskulatur ohne Betheiligung des Nervensystems rhythmische Contractionen auszuführen vermag, zu überzeugen. Scheinbare Unterstützung findet dieser beharrliche Wunsch einiger Untersucher in folgenden anatomischen und physiologischen Erscheinungen. Der Bau der Herzmuskulatur erscheint, wenigstens bei höheren Thieren und beim Menschen, um Einiges complicirter als solcher der Skelettmuskulatur. Einige Gifte verhalten sich in ihrer Wirkung auf die Herzmuskulatur anders als auf die Muskeln anderer Organe. Bis in die Gegenwart leistet man der Meinung Vorschub, dass es Thiere gebe, deren Herzen weder Nervenzellen noch Nerven enthielten, während solche Herzen sich doch rhythmisch contra-

1) Elementa Physiologiae. 1754. 
hirten; ebensolche Contractionen führe die abgeschnittene Spitze des Froschherzens unter gewissen Bedingungen aus. Es wird die Thatsache bervorgehoben, dass das embryonale Herz, in welchem noch keine Nervenelemente zu unterscheiden sind, sich auch rhythmisch zu contrahiren vermag.

In letzterer Zeit bestreiten L. Krehl und E. Romberg ${ }^{1}$ ) aus entwicklungsgeschichtlichen Gründen und sich auf ihre Versuche am Kaninchenherzen basirend, die Betheiligung der Nervenganglien an den automatischen Herzbewegungen und der Regulirung der Herzthätigkeit: „Nach ihrer Entwicklungsgeschichte schienen sie weder automatische Centren der Herzbewegung, noch Vermittelungsorgane der Vagus- oder Acceleranswirkung zu sein."

Allein die soeben angeführten Data sind entweder nur theilweise richtig, oder aber zweifelhaft, also sorgfältiger Controle bediirftig, oder sie sind endlich ganz falsch. Wie in der Mathematik ist in den Naturwissenschaften das endgiltige Resultat der zu lösenden Aufgabe in dem Marsse richtig oder falsch, in welchem die zum Auffinden des Unbekannten gegebenen Grössen richtig oder falsch waren. Eine Antwort erhält man wohl in beiden Fällen, dieselbe ist jedoch nur im ersteren Falle wahr; im letzteren Falle aber wird trotz des sorgfältigsten und regelrechten Ganges der Lösung ein falsches Resultat erzielt. Genau ebenso müssen die zur Erlangung einer endgiltigen, walrheitsgemässen Antwort in Bezug anf die von A. Haller vertretenen Theorie der Herzbewegungen herbeigezogenen Daten auf genauen anatomischen and physiologischen Thatsachen, gleichviel ob diesclben vom Autor selbst oder aus den Arbeiten anderer stammen, gegrtundet sein. Widrigenfalls können die erlangten Resultate, so zahlreich die Versuche und so sorgfältig ihre Ausführung sein möge, nicht auf Genauigkeit Anspruch machen und die Wissenschaft bereichern.

Solche Resultate nähern uns nicht der Wahrheit, sondern, ganz in Gegentheil, entfernen uns von ihr und hemmen die Entwicklung des wissenschaftlichen Wissens. So erscheinen mir

1) Ueber die Bedeutung des Herzmuskels und der Herzganglien für die Herzthätigkeit des Säugethiers. Archiv f. experim. Pathol. u. Pharmakologie. Bd. 30, p. 49. 
auch die von Krehl und Romberg erhaltenen Resultate, in Bezug auf die Bedentung der Muskulatur und der Ganglien des Herzens für die Thätigkeit des letzteren, mehr als zweifelhaft, weil sie von solchen anatomischen Daten ausgehen, welche nicht allein unrichtig sind, sondern auch schon lange vom wissenschaftlichen Standpunkte aus jedes Interesse verloren haben. Krehl und Romberg behaupten (p. 62), dass die Herzspitze des Frosches weder Ganglien noch Nerven enthalte („Froschherzspitze bekanntlich nicht nur Ganglien, sondern auch nervenfrei sein soll ${ }^{4}$ ). Nun ist es aber bekannt, dass in der oberen und unteren Hohlvene, im Venensinus, in den Vorhöfen, auf der Vorhofscheidewand, im Ventrikel, in der Ventrikelspitze und im Bulbus aortae des Froschherzens eine solche Menge von Nerven enthalten sind, dass es schwer wird zu sagen, ob mehr Muskelelemente oder mehr Nerven an der Zusammensetzung des Herzens überhaupt und der Herzspitze insbesondere sich betheiligen ${ }^{1}$ ).

Bezüglich der Vertheilung der Ganglien im Herzen des Frosches und der Säugethiere sind Krehl und Romberg, wie es scheint, die Untersuchungen von Joh. Dogiel ${ }^{2}$ ) und A. Kasem-Beck ${ }^{3}$ ) unbekannt geblieben. Joh. Dogiel hat Gruppen von Nervenzellen im oberen Drittel des Ventrikels, unterhalb der Atrioventrikularklappen, und einzelne Nervenzellen unterhalb des oberen Drittels des Ventrikels beschrieben (vergl. Fig. 4 u. 5 des cit. Journals). A. Kasem-Beck hat im Laboratorium von Joh. Dogiel das Herz vom Schaf, Kalb, Hunde, Ferkel, von der Ente, Truthenne und vom Huhn untersucht und längs des Sulcus longitudinalis anterior beim Schafherzen Ganglien in einer Entfernung von $25 \mathrm{~mm}$ von der Ventrikelbasis und einzelne Nervenzellen in einer Entfernung von $35 \mathrm{~mm}$ gefunden. In der hinteren Längsfurche traf er Gruppen von Nervenzellen in einer Entfernung von 20-25 mm von der Ventrikelbasis und einzelne Nervenzellen auf dem linken Ventrikel den Nerven entlang $(10 \mathrm{~mm}$

1) J. Dogiel und Tjumianzew, Zur Lehre über das Nervensystem des Herzens. Arch. f. mikrosk. Anat. Bd. 36.

2) J. Dogiel, Die Nervenzellen des Herzventrikels beim Frosche. Arch. f, mikrosk. Anat. Bd. 21.

3) A. Kasem-Beck, Ueber das Vorkommen von Ganglien und einzelnen Nervenzellen auf Herzventrikeln des Menschen, der Săuge thiere und der Vögel. Centralbl. f. med. Wiss. 1887, No. 42. 
weit von der hinteren Längsfurche) in einer Entfernung von $50 \mathrm{~mm}$ von der Ventrikelbasis. Die Länge des zur Untersuchung dienenden Schafherzens, von der Basis bis zur Spitze, betrug $75 \mathrm{~mm}$. Ausserdem fand Kasem-Beck auf dem Herzventrikel vom Kalbe, Hunde und Ferkel Gruppen von Nervenzellen in beträchtlicher Entfernung von der Ventrikelbasis. Wenn dieser Autor auch keine Gelegenheit hatte, in dieser Hinsicht noch das Kaninchenherz zu untersuchen, so wird wohl kaum die Structur und die Function des Herzens bei diesem Thier sich von der der von ihm untersuchten übrigen Säugethiere stark unterscheiden. Es ist also unverständlich, weshalb Krehl und Romberg keine Nervenzellen auf der Ventrikeloberfläche des Kaninchenherzens finden konnten. ( Keine Ganglien besitzen die Kentrikel, abgesehen von den vereinzelten Zellen am oberen Rande des Conus arteriosus. Insbesondere ist die Ventrikelscheidewand und also auch die Stelle, an der Kroncker und Schmey ein Coordinationscentrum suchten, frei von Ganglien.") In unserem Zweifel werden wir noch dadurch bestärkt, dass Krehl und Romberg auch die sebr eingehende Untersuchung von Vignal') über den Ganglienapparat der Säugethiere überhaupt und des Kaninchens speciell unbekannt geblieben ist. Sagt doch Vignal (p. 241) unter Anderem: "A la partie supérieure de ce plexus (plexus cardiaque proprement dit?), juste au-dessous des artères et des veines coronaires et par conséquent à la base même da ventricule, on trouve d'autres ganglions formés seulement de quelques cellules, ils sont généralement situés au-dessous du péricorde viscerale." Auch Vignal ist der Meinung (p. 243), dass kein grosser Unterschied in der Vertheilung des Nervenapparates im Herzen der Katze, des Hundes, Schafes und Kaninchens besteht (,jai trouvé chez tous ces animaux une disposition de cet appareil peu différente de celle qui existe chez le lapin“). Derselbe Vignal hat ferner den Nervenapparat beim Affen (Macacus simicus) untersucht, wobei er zur folgenden Schlussfolgerung bezüglich der Nervenzellen des Herzens bei demselben gelangte (p. 246): „Sur le tiers supérieur de ce plexus (les branches des plexus

1) Recherches sur l'appareil ganglionaire du coeur des vertébrés (Laboratoire d'histologie du Collège de France). Travaux de l'année 1881 publiés sous la direction L. Ranvier. 
coronaires), principalement sur les petites branches, on rencontre, outre les ganglions superficiels déjà décrits par Remak, un nombre considérable d'autres plus petits, qui die viennent de moins en moins abondants à mesure que l'on s'approche de la pointe du coeur, et qui disparaissent presque totalement, environ au point de naissance du deuxième tiers du ventricule. J'ai dit presque totalement, car les nerfs proches des gros vaisseaux partent des ganglions sur toute la moitié supérieure du ventricule."

Der Zweek der vorliegenden Abhandlung ist jedoch keine fingehende Erörterung aller von den Nachfolgern der A. Haller'schen Theorie zur Bekräftigung der selbstständigen rhythmischen, vom Nervensystem unabhängigen Herzcontractionen angeführten Hinweise. Dieser Artikel soll nur einige anatomisch-physiologischen Thatsachen in Bezug auf das Herz des Flusskrebses (Astacus fluviatilis), das längere Zeit hindurch als Paradigma eines von Nervenzellen und Nerven freien Herzens diente, bringen.

A. Brandt ${ }^{1}$ ), welcher im Laboratorium von S. Setschenow den Einfluss physikalischer und chemischer Reize auf das Her" des Flusskrebses untersucht hat, ist bezüglich der Herznerven dieses Thieres zu keinen positiven Resultaten gelangt. (,Ueber die Herznerven scheint nichts Näheres bekannt zu sein.")

Es weist auf Literaturangaben hin, welche aber nur die Voraussetzungen einiger Autoren enthalten (von C. Carus²), Newport ${ }^{3}$ ), Warnek ${ }^{4}$ ), Haeckel $\left.{ }^{5}\right)$ ). Uebrigens giebt A. Brandt die Möglichkeit zu, dass die Herznerven des Krebses von Nervus stomatogastricus ihren Anfang nehmen. (,Demnach wäre es nicht unwahrseheinlich, dass das Herz sowohl vom Stomatogastricus, als auch von der Bauchkette mit Nerven versorgt

1) A. Brandt, Physiologische Beobachtungen am Herzen des Flusskrebses. Mélanges biologiques de l'Academie des sciences de St. Pèterbourg. T. V (1865-66).

2) C. Carus, Von den äusseren Lebensbedingungen der heissund kaltblütigen Thiere. Leipzig 1824. (4. Beilage, No. 2, Ueber Herzschlag und Blut der Weinbergschnecke und des Flusskrebses.)

3) Newport, On the nervous system of the Sphinx ligustri. Philosop. transactions. 1832-1834.

4) Warnek, Leber des Flusskrebses. Diss. St. Petersburg. 1847 (russisch).

5) Haeckel, Ueber die Gewebe des Flusskrebses. Müller's Archiv. 1857. 
wird"). Ausserdem finden wir bei A. Brandt angegeben, dass es Jarschinski im Laboratorium von Prof. F. Owsjannikow missgliickt sei, Nervenganglien im Herzen des Flusskrebses nachzuweisen.

Weiter hat A. Meyer ${ }^{1}$ ), wie Jarschinski, sich vom Vorhandensein der Nervenganglien im Herzen des Flusskrebses nicht überzeugen können. Auch Eckhard ${ }^{2}$ ) erhielt negative Resultate in Bezug auf die Nervenganglien des Herzens von Cancer pagurus, ungeachtet dessen, dass es ihm gelungen war einen Nerven, welcher auf das Herz des von ihm untersuchten Thieres ähnlich dem Vagus bei anderen Thieren einwirkte, nachzuweisen. Durch Reizung dieses Nerven konnte Eckhard zwei Minuten lang dauernden Stillstand des Herzens herbeiführen.

Endlich erschien 1890 die prachtvolle Monographie von G. Retzius ${ }^{3}$ ) über das Nervensystem der Krebse, in welcher leider gar nichts über die Nervenzellen und Nerven des Herzens vom Flusskrebse zu finden ist. Nur auf der letzten, XIV. Tabelle, welche aber auch nicht den Ganglien und Nerven vom Flusskrebs (Astacus fluviatilis) gewidmet ist, finden sich zwei Abbildungen über Nervenendigung im Herzen - in der Muskulatur desselben -, von Palamon squilla; doch ist auch hier nichts von Nervenzellen des Herzens bei diesem Thiere erwähnt.

Der Autor dieser Zeilen konnte sich, einige von C. Carus und A. Brandt angeführten physiologischen Thatsachen in Betracht ziehend, nicht der Zweifel entwehren, ob auch die Angaben über den negativen Befund bezüglich der Ganglien und Nerven im Herzen der Crustaceen richtig seien. Dieser Zweifel wurde durch folgenden Umstand noch verstärkt: im Herzen von Thieren, welche auf einer niedereren Entwicklungsstufe als der Flusskrebs stehen, im Herzen von Insecten (Corethra plumicornis) sind von J. Dogiel ${ }^{4}$ ) Nervenzellen nachgewiesen worden. Dazu kommt

1) A. Meyer, Das Hemmungsnervensystem des Herzens. Berlin 1869.

2) Eckhard, Beiträge etc. IV. 1867.

3) G. Retzius, Zur Kenntniss des Nervensystems der Crustaceen. Stockholm 1890. Mit 14 Tafeln.

4) J. Dogiel, Anatomie und Physiologie des Herzens der Larve von Corethra plumicornis. Memoires de l'Academie des sciences de St. Péterbourg. 1877. 
noch, dass nicht jedes negative Resultat zur Lösung einer bestimmten Frage herbeigezogen werden kann: eine neue Untersuchungsmethode, oder eine und dieselbe, aber sorgfältiger und bewusster angewendete Methode kann bei der Erforschung einer Frage nicht selten ein negatives Resultat in ein positives verwandeln. Alles das veranlasste zur nenen Untersuchung des Baues und der Functionen des Herzens der Crustaceen.

J. Dogiel hat schon 1875 die Resultate seiner Untersuchungen über den Bau und die Funktionen des Herzen einiger Mollusken ${ }^{1}$ ) und Meerkrebse (Hummer, Languste und Krabbe) am Meeresufer des südlichen Frankreichs in einer kurzen Notiz an die Academie der Wissenschaften zu Paris ${ }^{2}$ ) über das Herz der Crustaceen niedergelegt. Einen eingehenderen Artikel über diesen Gegenstand hat er ein Jahr darauf in dem Journal für normale und pathologische Physiologie abgedruckt ${ }^{3}$ ).

Die Erforschung des Baues und der Funktionen des Herzens früher des Hummers, jetzt des Flusskrebses aus der Wolga bestand in einer makro- und mikroskopischen Untersuchung des Bates und der Funktionen dieses Organes: das freigelegte Her" wurde vor und während der mechanischen oder elektrischen Reizung, oder unter dem Einfluss der erhöhten oder herabgesetzten Temperatur und einiger Gifte direkt beobachtet.

Entfernt man die Schale des Flusskrebses, entsprechend der Lage des Herzens (Fig. 1, a), so kann eine rhythmische Bewegung einer bestimmten Stelle, welche von dem unter einer verhältnissmässig dicken und festen Haut befindlichen und sich contrahirenden Herzen abhängt, leicht wahrgenommen werden. Nach der Entfernung dieser Haut erscheint das Herz in der Form eines unregelmässigen Vierecks (Fig. 1, c), das am vorderen Ende etwas breiter als am hinteren ist; eine ebensolche Form besitzt das des Hummers. Die Verbindung des Herzens beim Flusskrebse mit Blutgefässen ersieht man aus Fig. 1, e, d. Fast von der ganzen Herzoberfläche gehen dünne Fäden aus, welche sich verschie-

1) J. Dogiel, Die Muskeln und Nerven des Herzens bei einigen Mollusken. Arch. f. mikrosk. Anatomie. Bd. XIV.

2) J. Dogiel, Compt. Rend. des séances de l'Acad. des sciences. Paris 1876.

3) J. Dogiel, Archives des physiol. norm. et pathologique BrownSéquard, Charcot et Vulpian. 1877. 
denartig durchflechten und hernach zu breiteren Bändern vereinigen und als Ligamenta cordis betrachtet werden können (Fig. 1, f und Fig. 2, c). Die obere und die untere Oberfläche des Herzens, oder richtiger gesagt, des Herzventrikels des Flusskrebses ist von einem verschieden geformten Epithel bedeckt (Fig. 3). Die untere Fläche des Herzens (des Ventrikels?) liegt auf einer dünnen, durchsichtigen Membran, welche den Ventrikel von der Leber, den Gesehlechts- und Verdauungsorganen trennt (Fig. 6). Diese Membram ist unter dem Namen Pericardialmembran (pericardium) bekannt, obwohl sie eher als Diaphragma bezeichnet werden könnte. An der Peripherie besteht sie aus stark ausgebildeten quergestreiften Muskelfasern, und in der Mitte aus einer sehnigen Ausbreitung (Fig. 6, b, a). Diese Membran, zusammen mit den anderen Theilen, welche den Herzventrikel umgeben, und der oben erwähnten Haut, welche ihn von oben bedeckt, umschliessen das Herz wie ein Sack, welcher schon mehr an einen Herzbeutel erinnert. Doch entspricht dieser Herzbentel, besonders sein unterer, unterhalb des Ventrikels befindliche Theil, seiner Funktion nach dem Vorhof anderer Thiere (atrium cordis), so dass am Herzen des Flusskrebses dieses Gebilde einerseits als Herzbentel, andererseits als Vorhof aufgefasst werden kann. Die innere und äussere Fläche des Herzbeutels - der oberen und unteren Membran - ist von einem flachen Epithel bedeckt (Fig. 3).

Ueber den Bau des Ventrikels und des Vorhofs vom Flusskrebsherzen lässt sich noch Folgendes sagen: $\mathrm{Zu}$ den Bestandtheilen des Herzens vom Flusskrebse gehören ausser dem seine äussere und innere Fläche bekleidenden Epitbel und dem Bindegewebe noch Muskeln, Nervenzellen und Nerven. Der Herzventrikel dieses Thieres enthält keine Blutgefässe als seine Ernährung vermittelnden Bestandtheil ; derselbe steht jedoch in Verbindnng mit der Arteria cephalica am Kopfende und mit der der Arteria sternalis und der Arteria abdominalis ihren Ursprung gebenden Arterie.

Das Herz der Crustaceen ist nach Milne Edward $s^{1}$ ) als ein Organ, welches zur Weiterbeförderung des arteriellen und nicht des venösen Blutes dient, aufzufassen. Bei dieser Weiterbeförderung des arteriellen Blutes durch das Herz des Flusskrebses

1) Leçons sur la physiologie et l'anatomie comparėe. 1858, p. 183. 
betheiligen sich die Oeffnungen im Ventrikel. Oben finden sich nach Milne Edwards zwei Paar Oeffnungen, unten ein Paar, vorn, zum Kopfe, und hinten zu je einer Oeffnung, zu Anfang der Arterien. Beim Flusskrebs sieht man thatsächlich nach der Eröffnung des den Ventrikel bedeckenden Pericardiums zum Kopfende hin zwei, an der unteren Ventrikelfläche befindlichen Oeffnungen durchschimmern.

E. Berger ${ }^{1}$ ) fand nach Behandlung mit Chlorgold oder Osmiumsäure und Isolation einzelne Nervenzellen nur in dem hinteren Theile des Herzens: "Ich habe sie blos im hinteren Theil des Herzens finden können."

In letzterer Zeit beschreibt Lydia Pogos chewa aus dem Laboratorium von Prof. F. Owsjannikow nicht allein einzelne Nervenzellen, sondern Ganglien, Gruppen von 4-6 Zellen, welche in dem durch die Muskulatur ziehenden Nervenstamm gelagert sind ${ }^{2}$ ). Doch konnte $\mathrm{P}$ og o s che wa im Herzen des Flusskrebses nicht mehr als nur einen Knoten auffinden.

Sowohl Berger wie $\mathrm{Pog}$ os chew a vergleichen die Nervenzellen im Herzen des Flusskrebses mit den Zellen des Abdominalknoten, doch sollen erstere etwas kleiner als letztere sein. Die Nervenzellen des Flusskrebsherzens beschreibt $\mathrm{P}$ o g o s c h e w a als ein - zwei- und dreipolare, ovale Zellen mit ganz rundem Kern und Kernkörperchen. Bei ihren Untersuchungen bediente sie sich der Vergoldungsmethode mit Zerzupfen und Schnitte nach vorhergegangener Erhärtung und nachträglichem Färben der Präparate mit Pikrokarmin.

Bei einer Controluntersuchung der Vertheilung der Nervenzellen und der Nerven im Herzen des Flusskrebses konnte ich mich überzeugen, dass im Herzen dieser Thiere nicht allein Nervenzellen, wie Berger glaubte, und nicht allein ein einziger Knoten, wie P o g o s c h e w a wähnt, sich vorfinden, sondern dass die Nervenzellen in eine der ganzen Länge des Herzens entlang ziehenden Kette, welche hauptsächlich aus zwei Gruppen, einer vordern und hintern (Fig. 7) besteht, wobei jede Gruppe aus

1) Ueber das Vorkommen von Ganglienzellen im Herzen vom Flusskrebs. Wiener Sitzungsber. d. k. Akademie d. Wiss. Bd. 74. 1877.

2) Die Nervenzellen der Scheeren und des Herzens vom Flusskrebs. Bote für Naturwissenschaften. St. Petersburg. 1890. No. 5 (russisch). 
mehreren Zellen (5-6) zusammengesetzt ist, angeordnet sind. Ausserdem finden sich einzelne Nervenzellen an anderen Stellen des Herzens. Die Hauptgruppen der Nervenzellen befinden sich in der Nähe der oben beschriebenen Oeffnungen und erinnern durch ihre Anordnung theilweise an die Ganglien von Remak und Bidder. Die Grösse und die Form der Nervenzellen ist eine ziemlich verschiedenartige; ihre Form ist mehr eine birnförmige (Fig. 12). Jede Zelle besteht aus dem Inhalt, Protoplasma und einem grossen Kern; sie sind uni- und multipolar, mit sich verzweigenden Fortsätzen, welche verschiedenartig zwischen den Muskeln verlaufen. Die bipolaren Nervenzellen erinnern ihrer Form nach an die Nervenzellen von Fischen (Fig. 8). Die Nervenzellen und Gruppen derselben sind zwischen den Bündeln der sich baumartig verzweigenden Nervenfasern (Fig. 7, 8, 9 und 15) gelagert.

Nicht ohne Interesse ist die Thatsache, dass die Nervenfaserbündel gegenseitig Fäserchen austauschen (Fig. 13), was an die Nervenanastomosen im Herzen der Frösche, der Säugethiere und des Menschen erinnert. Man stösst auch auf Nervenfasern, welche sich unter scharfen Winkeln theilen (Fig. 10). Eine solche Theilung geht besonders scharf an den Stellen, wo sich Nervenzellen befinden, vor sich.

Am caudalen Ende ist die Substanz unschwer nachzuweisen, welche unter dem Namen Punktsubstanz bekannt ist und derjenigen ähnelt, die schon in den Ganglien der Hauptnervenkette des Flusskrebses vorgefunden ( $\mathrm{Retzi}$ u s ?) wurde (Fig. 11). Die Muskeln des Herzventrikels vom-Flusskrebs gleichen ihrem Bau nach mehr den Herzmuskeln von Insekten (Corethra plumicornis), als solchem anderer Thiere, wie des Frosches, Kaninchens etc. In den Muskeln des Herzens wird kein Kern, wie in den Herzmuskeln der ubrigen Thiere sichtbar. Die Fasern der Muskelbündel zerfallen in feinste quergestreifte Fibrillen (Fig 4). Die Muskelbündel sind vom Bindegewebe umgeben.

Die das Herz von den Geschlechts- und Verdauungsorganen trennende und, wie erwähnt, als Vorhof aufzufassende Membran ist mit zahlreichen Nerven ausgestattet (Fig. 5), welche von der Bauchkette entspringen und theils im Vorhofe enden, theils auf den Ventrikel übertreten. Einige dieser Nerven gehen zum Ventrikel längs den Ligamenten des letzteren. Ueber den Verlauf 
dieser Nerven habe ich schon früher im Compt. rend. 1876 gesprochen: "Du ganglion situé chez la langouste, entre la deuxième et la troisième paires de pattes, il part des fibres nerveuses, qui se dirigent en haut et en dehors, puis viennent se diviser en partie dans les muscles voisins du pericorde, et s'unissent ensuite avec les muscles celni-ci." Indem ich hiermit die Beschreibung des Baues des Herzens vom Flusskrebse beende, muss ich noch einige Zeilen über die Untersuchungsmethode selbst, welche dabei zur Anwendung kam, hinzufügen.

Bei der Untersuchung der Nervenzellen und Nerven des Flusskrebses kamen zur Verwendung $2 \%$ Chlorgold- und $20 \%$ Ameisensäurelösung. Zuerst wurde das Herz in zwei Hälften zerschnitten, in eine obere und eine untere, und dann nach Möglichkeit so ausgespannt, dass eine möglichst grosse Oberfläche dieser Ventrikeltheile erst mit Ameisensäure- und darauf mit Chlorgoldlösung in Berïhrung gebracht werden konnte. Die ausgespannten Theile des zerschnittenen Ventrikels kamen zuerst auf 3-5 Minuten in die Ameisensäurelösung, wurden hierauf mit Wasser ausgewaschen und alsdann mit $2 \%$ Chlorgoldlösung $5-20$ Minuten hindurch behandelt, aufs Neue mit Wasser ausgespült und wieder in eine $20 \%$ Ameisensäurelösung gebracht, worin sie 12 und mehr Stunden verblieben. Die gefärbten, durch Ameisensäure aufgelockerten Ventrikeltheile kamen auf den Objektträger, wo sie mit Deckgläschen bedeckt wurden. Ein leichter Druck auf das letztere genügt nun, um das Präparat derart auszubreiten, dass bei geringer Vergrösserung (Hartn., Syst. 4, Ocul. 3) der Verlauf der Nervenstämme und die Vertheilung der Nervenzellen zwischen den Nervenfasern, in oben beschriebener Anordnung sehr deutlich zu sehen ist. Am schönsten wird das Gerüst von Nervenbündel und Nervenknoten erhalten, wenn man nach der Behandlung mit Chlorgold die Ventrikeltheile (oberer und unterer) in $20 \%$ Ameisensäurelösung 2--3 Tage liegen lässt und hierauf die aufgelockerten Muskelfasern mittelst Pinsel, unter beständiger Erneuerung des Glycerins, allmählich entfernt. Ausser Chlorgold habe ich auch $1 \%$ Osmiumsäurelösung angewendet. Der Verlauf der Nerven auf dem Pericardium konnte auch durch Methylenblau sehr deutlich gemacht werden. Zu diesem Zweck wurde eine Lösung desselben auf die unterhalb des Herzens befindliche Membran, nach Entfernung des letzteren, gebracht. Die 
Vertheilung der Nerven, welche nach der letzten Methode am Vorhof zur Anschauung kam, zeigt Fig. 5.

Aus dem über die Structur des Herzens beim Flusskrebs Gesagten geht hervor, dass dem Herz dieses Thieres Nervenzellen nicht abgehen, sondern im Gegentheil, dass dasselbe sehr reichlich mit Nervenzellen und Nervenzweigen ausgestattet ist.

Schon diese anatomischen Daten beweisen zur Genüge, dass diese Menge von Nervenelementen zwischen den Herzmuskeln des Flusskrebses nicht bei der Thätigkeit dieses Organs theilnahmslos bleiben können. Auch machen diese anatomischen Thatsachen uns verständlich, warum das aus dem Körper entfernte Herz des Flusskrebses fast eine Stunde lang sich zu contrahiren fortfährt. Selbst der in zwei gleiche Theile zerschnittene Ventrikel verliert nicht die Fähigkeit sich rhythmisch zu contrahiren: jeder Theil schlägt wie vordem. Die Untersuchung dieser Herzcontractionen des Flusskrebses nöthigt uns in dem Herz die Existenz einer seine Contractionen regulirenden Vorrichtung anzunehmen. Thatsächlich hat schon C. Carus ${ }^{1}$ ) in seinem Artikel über diesen Gegenstand eine Beobachtung, welche die Richtigkeit einer solchen Voraussetzung bestätigt, angeführt: nach dem Abschneiden des Kopfes beim Flusskrebs sah er Verlangsamung der Herzbewegungen sich einstellen; mechanische Reizung der Ganglienkette hatte eine ebensolche Verlangsamung und diastolischen Stillstand des Herzens zur Folge; zuweilen habe das Herz 5 Minuten lang still gestanden.

A. B rand $t^{2}$ ) spricht zum Schluss seines von uns citirten Aufsatzes, dass die Zerstörung des centralen Nervensystems des Krebses sowie die Eröffnung der harten Schale diastolischen Her'zstillstand herbeigeführt habe. Die direkte Reizung des Herzens mit inducirtem oder galvanischem Strome sei von Herztetanus begleitet gewesen, woraus er schliesst, dass das Herz beim Krebs eine Mittelstellung zwischen dem Wirbelthierherzen und den Skelettmuskeln einnehme.

Hierher gehören auch meine früheren, die Abhängigkeit der Herzcontractionen vom Nervensystem demonstrirenden Beobachtungen iiber die Herzfunction bei Meerkrebsen: „Lorsque chez

1) l. c.

2) 1. c. 
la langouste, le homard on d'autres crustacés, l'on excite le cordon ganglionnaire, on observe un ralentissement du coeur ou même son arrêt complet en diastole. On peut toujours provoquer un arrêt en diastole, si on irrite, au moyen de l'électricité, non seulement la chaîne ganglionnaire, mais encore le péricorde $\left.{ }^{4}{ }^{1}\right)$.

Meine neuerdings vorgenommenen Untersucbungen uber die Funktionen des Herzens beim Flusskrebs bestätigen sowohl die Angaben der mit dieser Frage beschäftigt gewesenen Forscher als auch meine früheren Betrachtungen am Meerkrebs. Reizt man mittelst Inductionsstrom die Ligamenta des Herzens beim Flusskrebs am vorderen oder hinteren Herzende, so erhält man diastolischen Herzstillstand, welcher 10-15 Secunden dauert. Durchschneidet man am vorderen (cephalen) oder hinteren (caudalen) Rande den Vorhof von oben, so stellt sich dabei zuweilen auch Herzstillstand ein. Am promptesten tritt diastolischer Herzstillstand auf und dauert am längsten, wemn die in Fig. 1 mit $\mathrm{h}$ bezeichnete, in der Mitte der das Herz von den Athmungsorganen trennenden Membran, in der Nähe der Muskeln $k, k$ befindliche Stelle mittels Inductionsstrom gereizt wird. Dieser Stillstand hängt davon $a b$, dass hier die Nerven zum Vorhof und dem Ventrikel verlaufen (Fig. 14). Zur Illustration des Gesagten seien hier einige Versuche angeführt.

Versuch I. Bei einem grossen Wolgakrebs wurde die harte Schale über dem Herzen entfernt, ohne die weiche den Ventrikel von oben bedeckende Haut zu verletzen. Das Herz schlägt hiernach 8-9 mal in 10 Secunden, zeitweilig 9 Secunden lang stillstehend. Reizung mittels Inductionstrom, bei einem mittelgrossen Grenet'schen Element und bei Spiralenabstand von $3 \mathrm{~cm}$ des Inductoriums von $\mathrm{G}$ a iffe, der in Fig. 1 mit $\mathrm{h}$ bezeichneten Stelle gab diastolischen Herzstillstand auf 40 Secunden. Nun wurde die das Herz von oben bedeckende Membran (ein Theil des Pericardiums) eröffnet. Das Herz schlug hiernach viermal in 10 Secunden. Neue Reizung derselben Stelle gab 40 Secunden lang dauernden Herzstillstand. Nach der Einstellung der Reizung fuhrte das Herz 9 Contractionen in 10 Secunden aus. Versuch II. Nach der Entfernung der harten Schale in der Herzgegend eines grossen Flusskrebses beobachtete man, wie

1) l. e. 
gewöhnlich, langdauernden Stillstand des Herzens im Diastole. Eröffinung der oberen, weichen Haut (des Pericardiums). Hierauf wurden 5 Herzschläge in 10 Secunden gezählt, dazwischen Pausen, welche bis 21 Secunden dauerten. Reizung der im Versuch I angegebenen Stelle - der Stelle durch welche die Nerven von der Bauchkette zum Herzen gehen -- mittels Inductionsstrom von derselben Stärke wie im vorhergehenden Versuch, rief diastolischen Herzstillstand, welcher 2 Minuten und 30 Secunden dauerte, d. h. während der ganzen Dauer der Reizung anhielt, herbei. Nach der Reizung schlug das Herz 2 mal in 10 Secunden und etwas später 13 mal in 10 Secunden. Eine neue Reizung mit demselben Strom hatte neuen Herzstillstand auf 22 Secunden zu Folge.

Versuch III. Nach der Eröffnung der harten Schale und des Pericardiums bei einem grossen Flusskrebs betrug die Zahl seiner Herzschläge 15 in 10 Secunden. Reizung derselben Stelle, wie in den vorhergehenden Versuchen, mittels Inductionsstrom, bei gleichem Spiralenabstand und demselben Gr e n e t'schen Element, gab 25 Secunden lang dauernden diastolischen Herzstillstand.

Die vorgefuhrten drei Beobachtungen zeigen, dass die Reizung mittels Inductionsstrom der Stelle, an welcher die extracardialen Nerven zum Herzen des Flusskrebses verlaufen, jedesmal einen diastolischen Herzstillstand von 22 bis 150 Secunden langer Dauer zur Folge hat. Nach der Einstellung der Reizung trat bald eine Beschleunigung der Herzcontractionen von 30 bis auf 78 Schläge in der Minute auf.

Wurden die Herzcontractionen des Krebses mittels eines empfindlichen Hebels, ähnlich demjenigen, welchen M a r e ${ }^{1}$ ) zum gleichen Zweck angewendet, verzeichnet, so erhielt man folgende Diagramme :

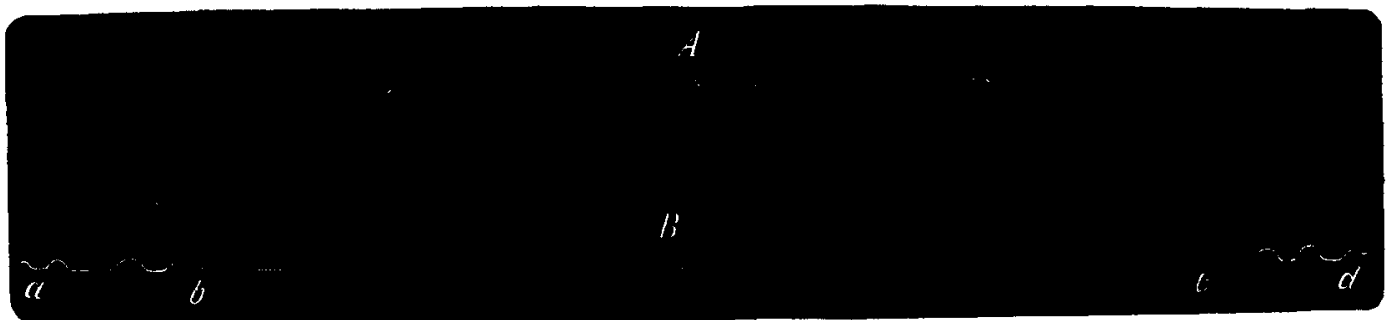

1) E. Marey, La circulation du sang a'l'état physiologique et dans les maladies. Paris 1881. 
Das Diagramm A zeigt normale Herzcontractionen des Flusskrebses. Das Diagramm B zeigt dasselbe Herz bis zur Reizung - $\mathbf{a} \mathbf{b}$-, während der Reizung der Hemmungsnerven - b cmittels Inductionsstrom bei $5 \mathrm{~cm}$ Spiralenabstand des $\mathrm{G}$ a iffeschen Inductoriums, und endlich nach der Reizung - c d. Der Herzstillstand währte 23 Secunden.

Diese Curven zeigen ebenfalls, dass die Reizung mittels Inductionsstrom der in oben angeführten Versuchen näher bezeichneten Stelle eine mehr oder weniger lange dauernden diastolischen Herzstillstand, welcher 15-30 und mehr Secunden währt, herbeiführt. Zur Reizung diente das Inductorium von Ga iffe bei einem mittelgrossen Gren et'schen Element.

Reizt man mittels Inductionsstrom das Herz (den Ventrikel) des Flusskrebses unmittelbar, so stellt sich bald, wie das Diagramm $\mathrm{C}$ uns vorführt, anstatt des diastolischen Herzstillstandes systolischer Herzstillstand - Tetanus - ein. Dieser Untersehied in der Wirkung der direkten Reizung des Herzens mittels Inductionsstrom beim Krebs und bei anderen Thieren erklärt sich meiner Meinung nach durch die verschiedene Struktur der Herzmusculatur bei ersterem im Vergleich zu der beim Froseh und bei den Säugethieren.

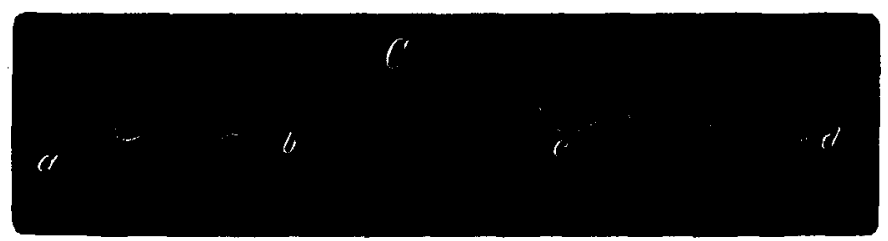

Das Diagramm C zeigt die Herzthätigkeit des Fusskrebses vor der Reizung - a b - während der direkten des Herzventrikels mittels Inductionsstrom, bei $5 \mathrm{~mm}$ Spiralenabstand des Induktoriums von $\mathrm{Ga}$ iffe - b c - und nach der Reizung c d. Aehnlichen Tetanus habe ich bei der direkten Reizmng des Hummerherzens beobachtet ${ }^{1}$ ).

Die Herzthätigkeit des Flusskrebses verändert sich nicht allein durch die Elektricitat oder höhere und niedere Temperaturgrade, sondern auch unter dem Einfluss einiger Gifte: Curare, Coffeïn, Aconitin, Digitalin ete. Es sei hier das Diagramm der

1) J. Dog'iel, l. c. 
Herzcontractionen unter dem Einfluss von Aconitin auf das Herz des Flusskrebses vorgeführt:

Das Diagramm I) zeigt normale Herzcontractionen des Flusskrebses und das Diagramm E - unregelmässige Contractionen Arhythmia - desselben Herzens unter dem Einfluss einer Lösung von deutschem Aconitin.

Somit sprechen anatomische und physiologische Thatsachen deutlich genug für die Abhängigkeit der Herzthätigkeit des Flusskrebses von im Herzen selbst befindlichen Nerven und Nervenzellen. Ausserdem ist die Struktur und die Funktionen des Herzens vom Flusskrebs in Vielem ähnlich, wenn auch nicht gleich, der Strucktur und der Funktion des Herzens von den abrigen Thieren.

\section{Erklärung der Abbildungen auf Tafel XII und XIII.}

Fig. 1. $a=$ harte Schale; $b=$ die den Herzventrikel (c) unmittelbar bedeckende Membran von Astacus fluviatilis; d, e, e = Blutgefüsse; $\mathrm{f}, \mathrm{f}=$ Ligamenta cordis; $\mathrm{g}=$ Athmungsorgane; $\mathrm{i}=$ knorpelartige, die Athmungsorgane von Geschlechts- und Verdauungsorganen und vom Herzen trennende Membran; $\mathrm{h}=$ Reizungsstelle der Nerven mittels Inductionsstrom; $\mathrm{k}, \mathrm{k}=$ Muskeln, durch welche die Nerven zum Ventrikel und zum Vorhof gehen; l, l= Klappen.

Fig. 2. $a=$ Ventrikel; $b=$ Klappen; $c, c=$ Ligamenta $d=$ Muskeln.

Fig. 3. Das die innere und die äussere, sowohl obere wie untere, Oberfläche des Ventrikels und des Pericardiums bekleidende Epithel.

Fig. 4. Ventrikelmuskulatur. Zeiss Syst. 4, Ocul. 12.

Fig. 5. Ein Theil des Vorhofs: $a=$ Muskeln; $b=$ Nerven. Hartn. Syst. 4, Ocul. 3. 
Beitrag zur vergleichenden Anatomie und Physiologie des Herzens. 239

Fig. 6. Membran, welche den Herzventrikel des Astacus fluviatilis von den Geschlechts- und Verdauungsorganen trennt - das Pericardium der Autoren, oder der Vorhof. $a=$ Sehnige Ausbreitung; $b=$ Muskelfasern.

Fig. 7. $a a=$ Zellengruppen in den Nervenbündeln b; Hartn. Syst. 2, Ocul. 2.

Fig. 8. Uni- (1) und bipolare (2) Zellen und Nerven (c). Hartn. Syst. 7, Ocul. 3.

Fig. 9. Nervenverzweigung zwischen den Muskeln des Herzventrikels (aa) und Nervenzellen (b, b). Hartn. Syst. 2, Ocul. 3; 'Tubus halb herausgezogen.

Fig. 10. Theilung der Nerven unter Winkelbildung (aa). Hartn. Syst. 7, Ocul. 3.

Fig. 11. Punktsubstanz. $a=$ Nervenfasern und $b=$ Nervenzellen.

Fig. 12. Nervenzellen mit langen Fortsätzen und mit Kernen in Herzen beim Flusskrebs. Zeiss Syst. 4, Ocul. 4; Tubuslänge $21 \mathrm{~mm}$.

Fig. 13. Nervenanastomose im Herzen von Astacus fluviatilis. Hartn. Syst. 2, Ocul. 2.

Fig. 14. Nerven (aa), welche von der Hauptkette durch die Muskeln (bb) in der Nähe des Herzens gehen und im Vorhof und im Ventrikel enden.

Fig. 15. Gruppen von Nervenzellen (aa) mit langen Fortsützen und Nerven.

Fig. 16. Nervenzellen aus dem Flusskrebsherzen mit Kern (b) und Kernkörperchen (a). Hartn. Syst. 7, Ocul. 3.

\section{Ueber Leimbildung aus Marksubstanz.}

Von

W. v. Nathusius, Halle.

In No. 336 v. 1890 des Zool. Anzeigers von Carus hatte ich eine Mittheilung uiber Leimerzeugung aus Marksubstanz von Federschäften gemacht. In der Kontroverse iber die Genesis der Marksubstanz war mir von beachtenswerther Seite nalıe gelegt, den Nachweis, dass die Marksubstanz Leimbildner sei, vollständiger zu führen, als bisher geschehen war, da dies als ein wichtiges Moment bezüglich der Frage, ob sic ein epidermoidales Gewebe sei, oder zn den Bindegeweben gehöre, betrachtet wurde. 


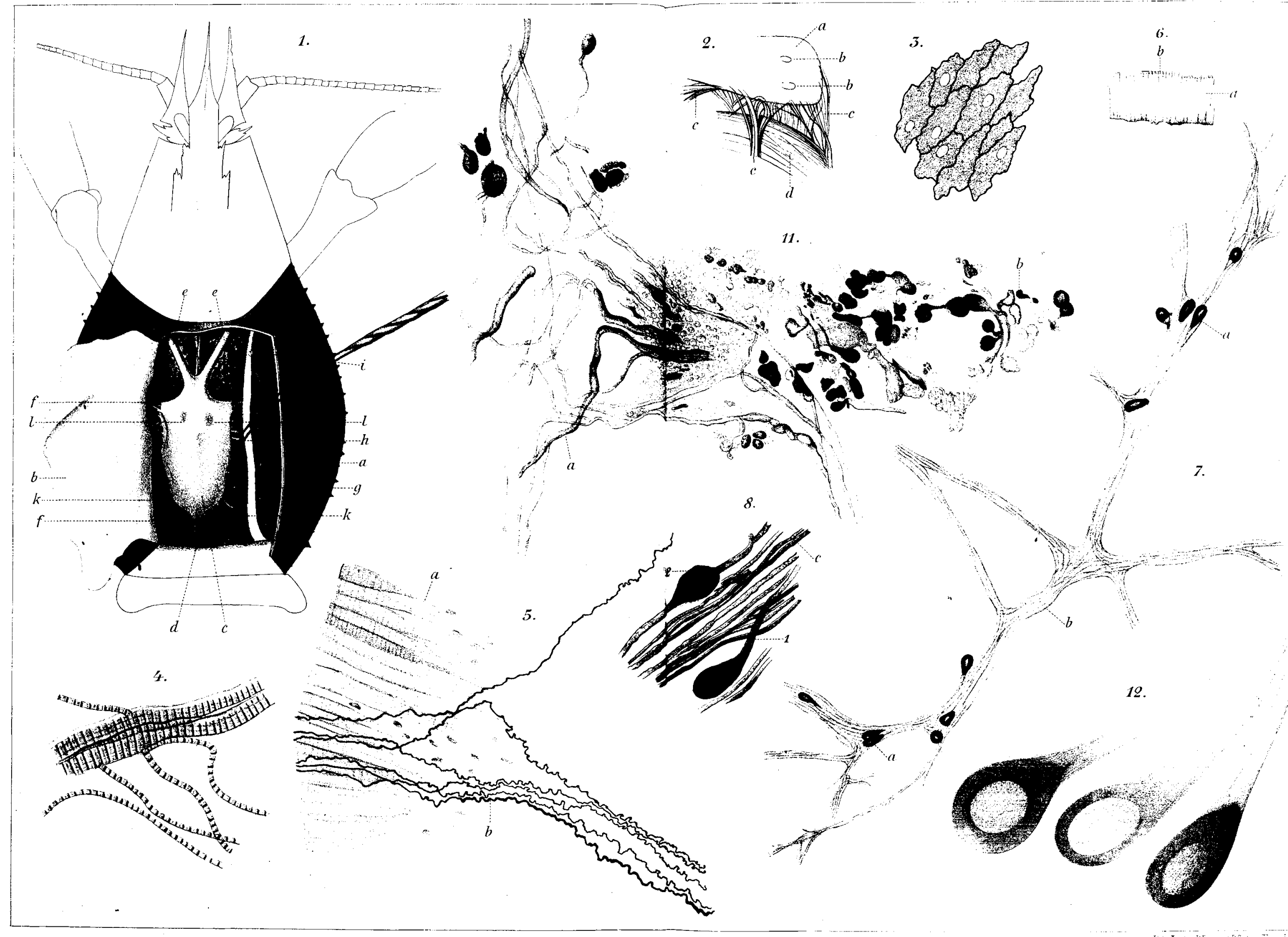






